\title{
Adverse Event Action Taken Relationship Delay Duration
}

National Cancer Institute

\section{Source}

National Cancer Institute. Adverse Event Action Taken Relationship Delay Duration. NCI

Thesaurus. Code C93706.

The time delay between the adverse event and the action taken. 